\title{
New Approaches to Arteriovenous Fistula Creation
}

\author{
Dheeraj K. Rajan, MD, FRCPC, FSIR ${ }^{1}$ \\ ${ }^{1}$ Division of Vascular and Interventional Radiology, University Health \\ Network, University of Toronto, Toronto, Ontario, Canada \\ Semin Intervent Radiol 2016;33:6-9
}

\begin{abstract}
Address for correspondence Dheeraj K. Rajan, MD, FRCPC, FSIR, Division of Vascular and Interventional Radiology, University Health Network, University of Toronto, 585 University Avenue, NCSB 1C-553, Toronto, Ontario, Canada M5G 2N2 (e-mail: dheeraj.rajan@uhn.ca).
\end{abstract}

\author{
Abstract \\ Keywords \\ - arteriovenous fistula \\ - dialysis \\ - creation \\ - surgery \\ - interventional \\ radiology
}

An autogenous arteriovenous fistula is considered the ideal access for hemodialysis delivery. However, surgical creation of an arteriovenous fistula is associated with less than optimal technical success, and multiple interventions are often required to assist maturation or maintain early patency. Given these shortcomings, multiple new approaches are now under investigation that possibly improve on surgical techniques and/or outcomes. Minimally invasive methods of creation with novel devices are under investigation, with preliminary published results available.
Objectives: Upon completion of this article, the reader will be able to identify recent advances in the creation of arteriovenous fistulae, including their technical considerations and obstacles to creating such fistulae.

Accreditation: This activity has been planned and implemented in accordance with the Essential Areas and Policies of the Accreditation Council for Continuing Medical Education (ACCME) through the joint providership of Tufts University School of Medicine (TUSM) and Thieme Medical Publishers, New York. TUSM is accredited by the ACCME to provide continuing medical education for physicians.

Credit: Tufts University School of Medicine designates this journal-based CME activity for a maximum of 1 AMA PRA Category 1 Credit $^{\mathrm{TM}}$. Physicians should claim only the credit commensurate with the extent of their participation in the activity.

In 1966, Brescia and Cimino described a surgical technique that has ultimately impacted millions of people worldwide: the surgical creation of the autogenous arteriovenous fistula (AVF). ${ }^{1}$ Despite this surgical innovation, vascular access remains the "Achilles Heel" of hemodialysis. ${ }^{2}$ It is the leading cause of hospitalization for patients undergoing hemodialysis, and is responsible for $\$ 2.9$ billion annual costs in the United States alone. ${ }^{3}$
Although an AVF is the recommended access due to its associated low mortality, morbidity, and cost compared with other access types, ${ }^{4-7}$ following AVF creation between 20 and $60 \%$ does not successfully mature or are rendered unsuitable for hemodialysis. This makes patients to prolonged catheter dependency with its associated morbidity and mortality. ${ }^{8-10}$ In addition, AVFs require an average of 1.5 to 3.3 interventions to facilitate usability for hemodialysis. ${ }^{11-13}$ Ultimately, the combination of these factors increases patient reluctance to undergo surgical fistula creation, particularly in individuals with previously failed fistulas. ${ }^{14}$ For these reasons, there is a clear and immediate demand and need for less invasive and more durable hemodialysis AVF options.

Various ideas and devices have been proposed over the years, with limited or no success, to address the concerns related to surgical AVF creation. However, novel technologies introduced recently have the potential to change how autogenous fistulas are created and to improve their durability.

\section{Optiflow Device (Bioconnect)}

The Optiflow device (Bioconnect Systems, Fort Washington, PA) is a prosthetic implant that is an anastomotic conduit from the artery to the vein. It is composed of nonthrombogenic siliconized polyurethane material, and has an angulated 10.1055/s-0036-1571805. ISSN 0739-9529. 
design used to standardize the surgical anastomosis and to optimize flow through this area ( - Fig. 1). Insertion requires surgical exposure of the artery and vein with insertion of the device into the brachial artery followed by placing the end of the divided cephalic vein onto the other portion of the device. The potential advantage of this device is standardization of the anastomosis and flow characteristics, thereby removing technical variability and potentially improving outcomes.

A prospective controlled pilot study was published in $2015{ }^{15}$ Forty-one patients underwent device placement with 39 matched controls. The primary endpoint was unassisted fistula maturation; patients were observed for 90 days. Additionally, safety from adverse events was documented. The ability to support dialysis was not assessed. At 90 days, unassisted maturation with the Optiflow device was 68\% compared $76 \%$ for the control group $(p=0.47)$, with no device-related serious adverse events. Acute thrombosis or fistula failure was noted in 7\% (3/41) of device cases. The study was not powered to assess differences in patency and maturation between groups. ${ }^{15}$ A larger investigational study is underway within the United States to assess clinical efficacy of this novel device (clinicaltrails.gov; NCT01921933).

\section{Human Pancreatic Elastase}

By applying human pancreatic elastase (HPE) at the time of surgery, the hope is that HPE will assist with fistula maturation and overall patency. Pig pancreatic lipase has been shown to result in elastin fragmentation and decreased intimal hyperplasia when applied to the surface or adventitia of blood vessels. ${ }^{16}$ Proteon Therapeutics (Waltham, MA) has introduced PRT-201, a type 1 recombinant pancreatic elastase that is applied once to the outside of vessels at the time of AVF creation.

A randomized, double-blinded study of PRT-201 was performed on patients undergoing radiocephalic or brachiocephalic AVF creation to assess safety and efficacy of the

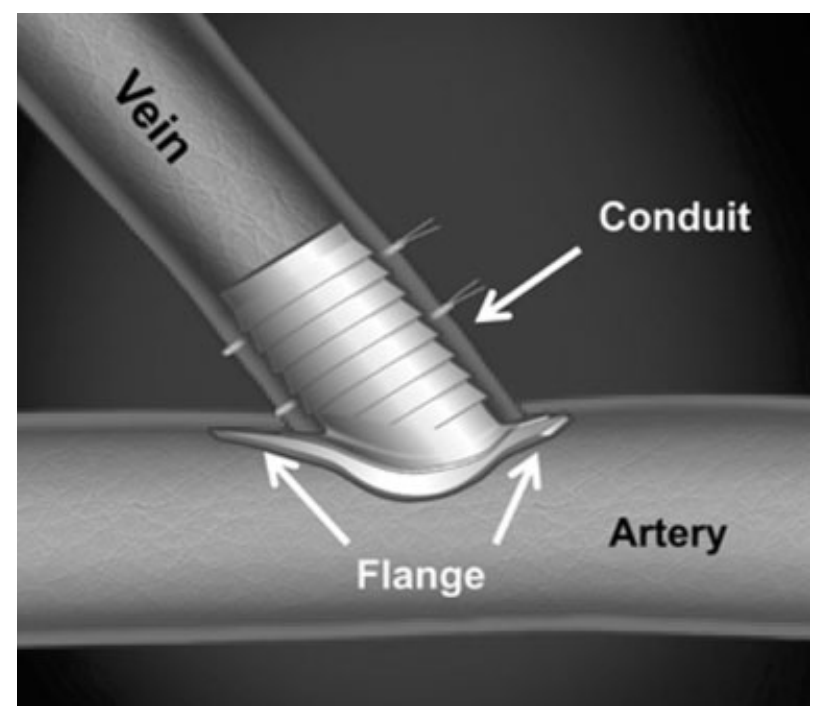

Fig. 1 Optiflow device (used with permission, Bioconnect Systems, Fort Washington, PA). product, which was measured as unassisted fistula maturation. Immediately following creation of the AVF, the PRT-201 solution (in two different doses of 10 and $30 \mu \mathrm{g}$ ) or placebo (saline) was dripped onto the exposed inflow artery, anastomosis, and outflow vein. No difference in adverse events was noted between the treatment and placebo groups. The primary efficacy measure of unassisted primary patency was not significantly different between groups. However, PRT-201 use was associated with improved unassisted maturation at 3 months (67\%, 87\% [ $p=0.03$ ], and 92\% [ $p<0.01]$ for the placebo, 10 , and $30 \mu \mathrm{g}$ dose groups, respectively). The authors concluded that PRT-201 administered at the $30 \mu \mathrm{g}$ dose may be most effective for radiocephalic fistulas, but larger studies are required to validate this observation. ${ }^{17}$

\section{Bioengineered Blood Vessels}

In patients lacking suitable veins for AVF creation, bioengineered veins constructed from cultivated donated human cells on a tubular scaffold can be surgically placed (-Fig. 2). The tissue is a decellularized collagen structure that does not trigger an immune reaction. The efficacy for providing durable AVF access is currently being assessed in a clinical trial sponsored by Humacyte Inc. (Morrisville, NC). Sixty patients are currently enrolled (20 in the United States, 40 in Poland) in a safety and efficacy trial, with efficacy determined by patency at 6 months (clinicaltrials.gov; NCT01744418). Following this preliminary study, a pivotal, global trial of this bioengineered vessel for use in vascular access creation is forthcoming.

\section{Minimally Invasive Creation of Arteriovenous Fistulas}

For any interventionalist performing hemodialysis interventions, the possibility of creating an AVF percutaneously has remained an elusive goal. In theory, creating a fistula without traditional open surgery may reduce vessel trauma, thereby reducing the stimulus for intimal hyperplasia that is

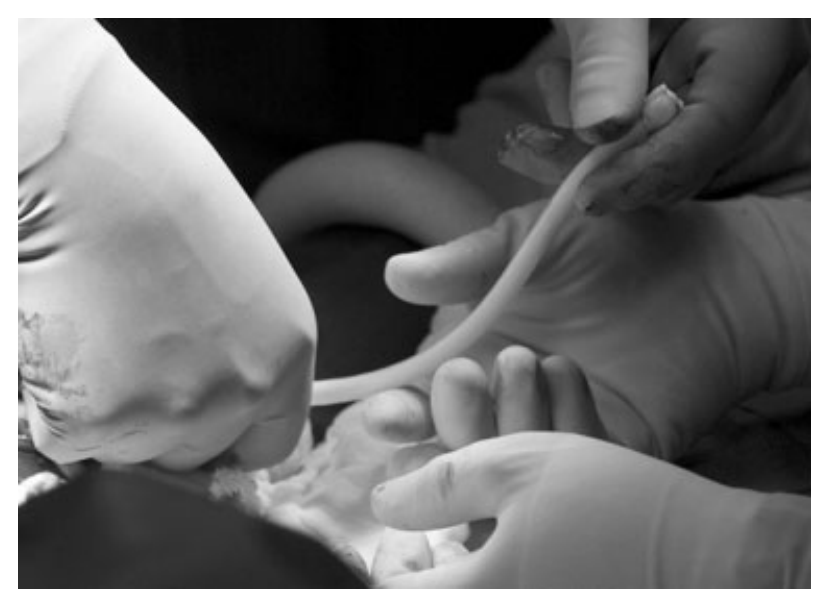

Fig. 2 Bioengineered vein being surgically inserted (Courtesy of Dr. Lawson, Duke Medicine). 

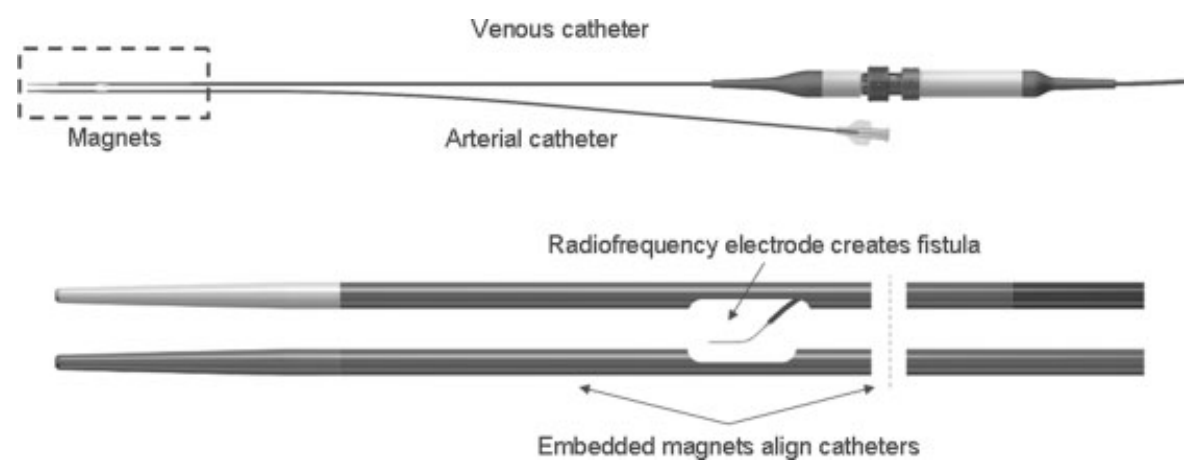

Fig. 3 EverlinQ endoAVF System (TVA Medical Inc., Austin, TX) (This device has not been studied in the United States and has not been approved for commercial use by the United States Food and Drug Administration).

associated with fistula maturation failure, ${ }^{18}$ with concomitant reduced morbidity and improving patient acceptance and fistula use. Within the past couple of years, the possibility of percutaneous AVF (pAVF) creation has become a reality. There are currently two devices under investigation.

\section{everlinQ endoAVF System}

The everlinQ endoAVF system (TVA Medical Inc., Austin, TX) consists of two $6 \mathrm{~F}$ catheters that contain rare earth magnets (-Fig. 3). Under fluoroscopy, one magnetic catheter is inserted into the ulnar artery, and the other magnetic catheter is inserted into the ulnar vein. When the catheters are aligned with one another, the magnets within each catheter are attracted, juxtaposing the artery and vein, while simultaneously aligning a radiofrequency electrode in the venous catheter and a ceramic backstop in the arterial catheter. The radiofrequency electrode is then released from the venous catheter and energized for 2 seconds, creating an approximate $5 \mathrm{~mm} \times 1 \mathrm{~mm}$ channel between the ulnar vessels. This creates a side-to-side ulnar artery to ulnar vein fistula. No prosthetic material is left at the fistula site (-Fig. 4).

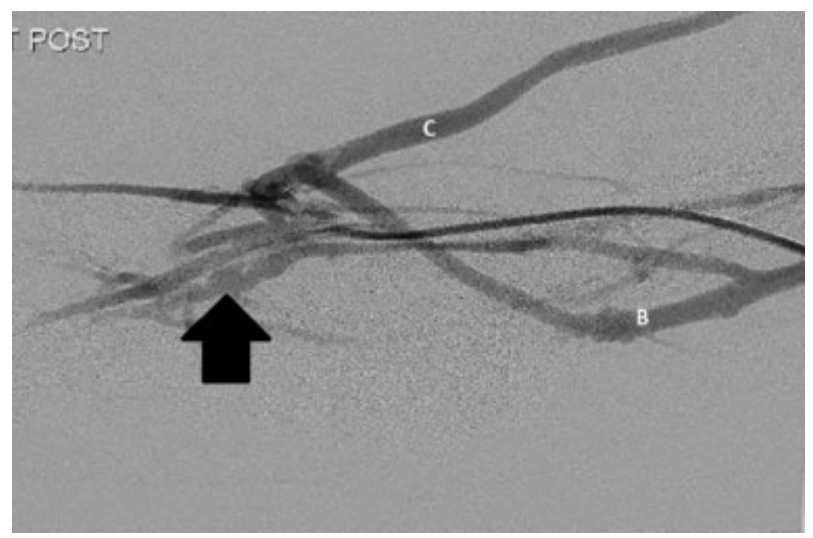

Fig. 4 EndoAVF (TVA Medical Inc., Austin, TX) created between the ulnar vein and ulnar artery (arrow). Injection of contrast from the brachial artery demonstrates flow through the fistula filling the cephalic vein (C) and the basilic vein (B).
The FLEX study was a prospective study that evaluated this technology. The study enrolled 33 patients and reported a $97 \%$ technical success rate, 58 days to maturation, and $96 \%$ of pAVFs were considered mature (via Duplex ultrasound) and usable for dialysis. ${ }^{19}$ of the patients who completed follow-up, 96\% were able to undergo hemodialysis during the 1-month follow-up period. This study validated the feasibility of creating a usable AVF percutaneously, with results that surpassed historical surgical outcomes.

Building on this initial study, a larger multicenter study has completed recruiting 80 patients in Canada, Australia, and New Zealand, and is currently in the follow-up phase. The primary efficacy endpoint being assessed is the percentage of patients with fistula maturation/usability (clinicaltrials.gov; NCT02036671).

\section{Ellipsys Device}

Another device (Ellipsys; Avenue Medical, San Juan Capistrano, CA) is currently enrolling up to 105 patients under an investigational device exemption (IDE) Study in the United States (clinicaltrials.gov; NCT02363972). The primary outcome measure is maturation rate, defined as percentage of fistulae suitable to allow successful cannulation for dialysis within 90 days. The device is a single unit that engages the arterial and venous walls; a fistula is created with low-power direct current energy. The AVF is created in the forearm between a perforating vein and the radial artery. In an initial study, results were presented at the Society of Interventional Radiology Meeting in 2014. Ten patients underwent fistula creation with the Ellipsys device, with a $100 \%$ technical success and $70 \%$ clinical success defined as ability to dialyze for one session. ${ }^{20}$ Further larger scale studies are needed.

\section{Conclusion}

In recent years, many new technologies have been introduced to overcome less-than-ideal AVF surgical outcomes. Many of these technologies are adjuncts to surgical creation, yet viable minimally invasive options now exist with promising published data that offer an alternative to traditional surgical AVF creation. 


\section{References}

1 Brescia MJ, Cimino JE, Appel K, Hurwich BJ. Chronic hemodialysis using venipuncture and a surgically created arteriovenous fistula. N Engl J Med 1966;275(20):1089-1092

2 Schwab SJ. Hemodialysis vascular access: the Achilles' heel remains. Kidney Int 2007;72(6):665-666

3 Saran R, Li Y, Robinson B, et al. US Renal Data System 2014 Annual Data Report: Epidemiology of kidney disease in the United States. Am J Kidney Dis 2015;66(1, Suppl 1):S1-S305

4 Malas MB, Canner JK, Hicks CW, et al. Trends in incident hemodialysis access and mortality. JAMA Surg 2015;150(5):441-448

5 National Kidney Foundation-Dialysis Outcomes Quality Initiative. NKF-DOQI clinical practice guidelines for vascular access. Am J Kidney Dis 1997;30(4, Suppl 3):S150-S191

6 Kinney R; Centers for Medicare and Medicaid Services. 2005 Annual Report: ESRD Clinical Performance Measures Project. Am J Kidney Dis 2006;48(4, Suppl 2):S1-S106

7 Peters VJ, Clemons G, Augustine B. "Fistula First" as a CMS breakthrough initiative: improving vascular access through collaboration. Nephrol Nurs J 2005;32(6):686-687

8 Asif A, Roy-Chaudhury P, Beathard GA. Early arteriovenous fistula failure: a logical proposal for when and how to intervene. Clin J Am Soc Nephrol 2006;1(2):332-339

9 Dember LM, Beck GJ, Allon M, et al; Dialysis Access Consortium Study Group. Effect of clopidogrel on early failure of arteriovenous fistulas for hemodialysis: a randomized controlled trial. JAMA 2008;299(18):2164-2171

10 Al-Jaishi AA, Oliver MJ, Thomas SM, et al. Patency rates of the arteriovenous fistula for hemodialysis: a systematic review and meta-analysis. Am J Kidney Dis 2014;63(3):464-478

11 Biuckians A, Scott EC, Meier GH, Panneton JM, Glickman MH. The natural history of autologous fistulas as first-time dialysis access in the KDOQI era. J Vasc Surg 2008;47(2):415-421, discussion 420-421

12 Falk A. Maintenance and salvage of arteriovenous fistulas. J Vasc Interv Radiol 2006;17(5):807-813

13 Rooijens PP, Burgmans JP, YoTI, et al. Autogenous radial-cephalic or prosthetic brachial-antecubital forearm loop AVF in patients with compromised vessels? A randomized, multicenter study of the patency of primary hemodialysis access. J Vasc Surg 2005;42(3): 481-486, 487

14 Chaudhry M, Bhola C, Joarder M, et al. Seeing eye to eye: the key to reducing catheter use. J Vasc Access 2011;12(2):120-126

15 Nikam M, Chemla ES, Evans J, et al. Prospective controlled pilot study of arteriovenous fistula placement using the novel Optiflow device. J Vasc Surg 2015;61(4):1020-1025

16 Amabile PG, Wong H, Uy M, et al. In vivo vascular engineering of vein grafts: directed migration of smooth muscle cells by perivascular release of elastase limits neointimal proliferation. J Vasc Interv Radiol 2002;13(7):709-715

17 Hye RJ, Peden EK, O'Connor TP, et al. Human type I pancreatic elastase treatment of arteriovenous fistulas in patients with chronic kidney disease. J Vasc Surg 2014;60(2):454-461.e1

18 Lee T, Roy-Chaudhury P. Advances and new frontiers in the pathophysiology of venous neointimal hyperplasia and dialysis access stenosis. Adv Chronic Kidney Dis 2009;16(5): 329-338

19 Rajan DK, Ebner A, Desai SB, Rios JM, Cohn WE. Percutaneous creation of an arteriovenous fistula for hemodialysis access. J Vasc Interv Radiol 2015;26(4):484-490

20 Hull JE, Velez JH, Martinez JP. Percutaneous proximal radial artery arteriovenous fistula creation for hemodialysis using the Ellipsys $^{\mathrm{TM}}$ vascular access system. J Vasc Interv Radiol 2014;25 (3 Suppl):S20 